\title{
Medical errors: the importance of the bullet's blunt end
}

\author{
Piet Leroy
}

Received: 11 August 2010 / Accepted: 17 August 2010 /Published online: 1 September 2010

(C) The Author(s) 2010. This article is published with open access at Springerlink.com

Medical errors are substantial causes of hospital-related morbidity and mortality. It is estimated that preventable medical errors alone cause up to 98,000 annual deaths in the United States - the daily equivalent of one fatal jumbo jet crash [8]. Preventable adverse events and accidents impose a great burden on patients and on the health care system [5]. The finding that medical care in itself may generate harmsometimes fatal and sometimes persistent-has caused real shockwaves of worrying. The society is not likely to tolerate major accidents in a technologically high standing and expensive activity that is mastered by highly trained professionals and that is intended to heal and not to harm [1]. The adagium primum non nocere (first, do not harm) is one of the principal ethical precepts taught in medical school. However, both unpretentiousness as well as righteous realism point out that in medicine, like in any professional activity, adverse events and accidents may be part of daily practice. Ignoring the fact that also in medicine, Errare humanum est (to err is human) or remaining indifferent to its relevance is nothing less than a sign of dramatic irresponsibility.

Children may be particularly at risk for medical harm. Dependency, limited communication skills, immature anatomy and physiology, the need for age- or weight-adapted equipment, and the need for dosing calculations have all been suggested as potential risk factors [7]. In technically complex health care

P. Leroy

Division of Paediatric Intensive care, Department of Paediatrics, Maastricht University Medical Centre,

Maastricht, The Netherlands

P. Leroy $(\triangle)$

Department of Paediatrics, University Hospital Maastricht, P.O. Box 5800, 6202 AZ Maastricht, The Netherlands

e-mail: p.leroy@mumc.nl systems, children's vulnerability may still be more pronounced. In this issue, Niesse et al. report their analysis of critical incidents (CI) in severely ill children admitted to a Paediatric Intensive Care Unit (PICU) [6]. Prospectively collected (near) incidents were compared to retrospectively assessed patient data. By use of logistic regression analysis, mechanical ventilation, male gender, and length of stay were identified as significant predictors for the occurrence of (near) incidents. In theory, these finding are interesting, but their practical value remains limited. At first, the three independent predictors that were characterized can hardly be regarded as potential targets for improvement strategies. None of them can effectively be changed or avoided, except by a policy that prevents PICU admissions in general or intubations and invasive ventilation in particular. Recent advances in highflow oxygen therapy and non-invasive ventilation may partially help to prevent the latter, although these techniques will certainly be associated with their own risk profile. Second, there is no doubt that within a population of sick children, some will be more prone to CIs than others. Recognizing this difference may help in the triage of pediatric patients to the safest possible care. However, since any PICU patient is subject to complex medical care involving many professionals and technical interventions, they all must be considered as being de facto at risk for CIs. This makes it less interesting to identify more precisely those patients who have higher risks, unless one was able to divide the PICU population in, respectively, "no risk at all" and "at risk" subgroups. Since all PICU patients are at risk and individual risk profile may change over time, a CI preventive strategy should be applied to the total population and at all times.

Niesse et al. nicely illustrate the traditional "sharp-end" approach to medical errors by attributing them to directly observable factors on the spot of the actual accident. Although this may work for simple, straightforward systems, it will be 
insufficient to deal with errors occurring in highly complex systems like medicine. Causes of errors can be situated in individual patients and individual professionals but just as much in factors that are more distant or even unobservably far from the actual working context. Examples of these "blunt end"-situated factors are working conditions (e.g., availability and content of protocols, workload, fatigue due to excessive working hours, professional relations, communication, work atmosphere, importance of hierarchy, reluctance or resistance to report malpractice, etc.), the local policy (e.g., choices of material, lack of appropriate tools, and retrenchments in expenditure), and even legislation. In complex systems, also a "blunt-end" approach is needed in order to reveal the underlying system defect that allowed the opportunity for the error to occur.

A corner stone of quality improvement and error reduction in a "blunt-end" approach is the availability of a continuous and easily accessible tool for error reporting. Niesse et al. nicely illustrate the usefulness of a blame-free CI-reporting system in identifying incidence and characteristics of common CIs. Provider-reported data may have important eyeopening and problem-elucidating effects by offering the opportunities to understand factors that contribute to or prevent harm to patients. Error reporting may lead to the creation of customized interventions that effectively reduce the incidence of errors [2]. In order to make error reporting profitable, some essential preconditions should be fulfilled.

First, the reporting system should apply a low threshold. This means that also near incidents and minor incidents must be reported. Based on experience in high technological industries (e.g., oil industry and civil aviation), it is now widely accepted that it makes more sense to screen systematically for (the much more common) near incidents than to focus only on the analysis of obvious severe accidents. It has been shown that most preventive system changes come from minor incidents that occurred repeatedly over long periods [4]. In my own experience, handling a low threshold also helps professionals to gain experience in dealing with adverse events. This may create an open-debate culture in which reporting (near) incidents becomes an evident part of daily practice.

Second, error reporting should be blame-free. Traditionally, errors are "sharp-end-wise" attributed to individual mistakes, and blamed professionals may be subject to penalization. However, true negligence or guilty neglect is only rarely the major cause of a medical error. Errors need to be considered as system-based phenomena, which arede facto-impossible to eradicate completely. In order to make the complex medical system safer, it is therefore essential to uncover in time any defects that may lead to major accidents within the foreseeable future. A system that permits admitting errors honestly is more likely to achieve this goal, compared to a punitive approach [3]. Reporting errors should be encouraged as a sign of professionalism and responsibility. Blame-free reporting does not mean that professionals are released from the duty to deliver excellent medical care at all times. Neither should it be considered as an excuse for neglect or negligence.

Third, error reporting should be applied to all levels and professions involved in the patient care and in all possible directions. The most junior team member must be able to correct a senior consultant or to report errors that have been made by superiors, without running the risk of retaliation. This will often require proper teamwork and flattening of existing hierarchies.

Finally, error reporting cannot be an isolated activity. Reported errors must be discussed within a multidisciplinary surveillance group that analyzes the reported (near) incidents, identifies critical situations or processes, implements initiatives for improvement, gives systematic feedback to the frontline professional, and continuously generates incentives for effective reporting by professionals. In addition, health care authorities, both institutional as well as political, must be prepared to accept the consequences of a system-based approach of dealing with medical errors. The failure to improve blunt end-situated causes of medical errors will cause frustration amongst health care workers in the short term and certainly to patient harm in the long term.

Open Access This article is distributed under the terms of the Creative Commons Attribution Noncommercial License which permits any noncommercial use, distribution, and reproduction in any medium, provided the original author(s) and source are credited.

\section{References}

1. Begley S (2008) Go to the hospital at your own risk. Newsweek April 2008

2. Cimino MA, Kirschbaum MS, Brodsky L, Shaha SH (2004) Assessing medication prescribing errors in pediatric intensive care units. Pediatr Crit Care Med 5(2):124-132

3. Dickey J, Damiano RJ Jr, Ungerleider R (2003) Our surgical culture of blame: a time for change. J Thorac Cardiovasc Surg 126(5):1259-1260

4. Frey B, Buettiker V, Hug MI, Waldvogel K, Gessler P et al (2002) Does critical incident reporting contribute to medication error prevention? Eur J Pediatr 161(11):594-599

5. Miller MR, Zhan C (2004) Pediatric patient safety in hospitals: a national picture in 2000. Pediatrics 113(6):1741-1746

6. Niesse OW, Sennhauser FH, Frey B (2010) Critical incidents in paediatric critical care: who is at risk?. Eur J Pediatr 170:193-198

7. Skapik JL, Pronovost PJ, Miller MR, Thompson DA et al (2009) Pediatric safety incidents from an intensive care reporting system. J Patient Saf 5(2):95-101

8. Walsh AP, Baum JD, Walsh DJ (2010) Flight plans, patient safety and American hospitals - is it time to check the baggage of private sector oversight? J R Soc Med 103(6):215 